\title{
Effect of Neurofeedback on Visual-Spatial Attention in Male Children with Reading Disabilities: An Event-Related Potential Study
}

\author{
Neda Sadeghi ${ }^{1}$, Mohammad Ali Nazari ${ }^{*}$ \\ ${ }^{1}$ Department of Neuroscience, Iran University of Medical Science, Tehran, Iran \\ ${ }^{2}$ Department of Psychology, University of Tabriz, Tabriz, Iran \\ Email: ${ }^{*}$ alinazari@tabrizu.ac.ir
}

Received 23 April 2015; accepted 15 June 2015; published 18 June 2015

Copyright (C) 2015 by authors and Scientific Research Publishing Inc.

This work is licensed under the Creative Commons Attribution International License (CC BY). http://creativecommons.org/licenses/by/4.0/

\section{(c) (i) Open Access}

\begin{abstract}
Recent studies describe a number of difficulties associated with attention deficit in children with reading disabilities. Information about visual-spatial attention mainly arises from studies using event-related potentials (ERPs) during Posner's spatial cueing paradigm. This study aims to use neurofeedback with a special protocol for treating children with reading disabilities, and moreover, to evaluate visual-spatial attention ability by means of Posner paradigm task and ERPs. The study was conducted in a single subject design in 20 sessions. Participants were 2 male children, aged between 10 - 12 years old, who completed twelve 30-min neurofeedback sessions. Repeated measurements were performed during the baseline, treatment, and post treatment phases. Results showed some improvement in Posner paradigm parameters (correct response, valid and invalid reaction times). Furthermore, grand average ERPs for both of the participants in each of the four conditions (Valid-right, Invalid-right, Valid-left and Invalid-left) were analyzed. The analysis of P3 component showed a reduction in latency, indicating an improvement in the timing of cognitive processes. In addition, the graphs showed a decrease in amplitude level, which meant easier processing than before.
\end{abstract}

\section{Keywords}

Neurofeedback, Reading Disability, Visual-Spatial Attention, ERP, Event-Related Potentials

${ }^{*}$ Corresponding author.

How to cite this paper: Sadeghi, N. and Nazari, M.A. (2015) Effect of Neurofeedback on Visual-Spatial Attention in Male Children with Reading Disabilities: An Event-Related Potential Study. Neuroscience \& Medicine, 6, 71-79. 


\section{Introduction}

Reading disability (RD), the most prevalent form of learning disorder, is recognized essentially as a significant difference between an individual's reading ability and his or her achievement based on age, intelligence, education and social supports [1]. Although RD is one of the most common childhood disorders, prevalence is uncertain. Studies conducted in English-speaking countries show prevalence ranging from 5\% to 17.5\% [2]. This disorder is considered a cognitive dysfunction, itself secondary to a neurobiological dysfunction. Many researches in cognitive neuropsychology, neuroscience and genetics have resulted in suggestions for possible causes of dyslexia. Although this disorder might be secondary to more basic auditory temporal processing deficits or speech perception deficits, phonological deficit as the cognitive basis of developmental dyslexia is now widely accepted. In addition, low level visual processing disorders induced by a magnocellular dysfunction may contribute to developmental dyslexia [3].

Modern electrophysiologic and brain imaging technologies have used to enhance diagnostic precision and illuminate the neurobiological underlying of learning disorders [4]. Electroencephalography (EEG) provides methods by which human brain activity can be assessed during cognitive processes. Computerized EEG studies including evoked potentials (EP) or event-related potentials (ERP), spectral EEG analysis, and topographic EEG brain mapping have also identified a number of brain irregularities in individuals with learning disabilities [4]. Children with reading disability, in addition to reading problems, also have a number of difficulties associated to attentional deficits. Moreover, it has been reported that children with reading disability have impaired visual-spatial attention abilities [5]. Currently, findings suggest that phonological problems and the reading impairment in dyslexia both result from poor visual coding. More precisely, the retinal cells have an important role in serial scanning and spotlighting the letters of a text [6]. Vidyasagar et al. (2009) suggested that an early perceptual defect occurring in visual pathway was the cause of reading problems in children with dyslexia. They also discussed attentional mechanisms controlled by the dorsal visual pathway having a special role in serial scanning of letters. Any deficits in this process will result in subsequence effects, including problems in visual processing of graphemes, changing them into phonemes and the enhancement of phonemic awareness [7] [8].

Such information about visual-spatial attention mainly arises from studies using ERPs. ERPs were first recognized in 1964, and had since remained a diagnostic instrument in both the fields of psychiatry and neurology [9]. ERPs are those EEGs that directly measure the electrical response of the cortex to sensory, affective, or cognitive events [9]. In most ERP studies on visual-spatial attention, researchers often use a variant of Posner paradigm. In this paradigm, subjects should focus attention on one side and ignore events in the opposite side [10]. In this task, the subject is instructed to fixate at a central point on the screen. A target stimulus appears on either the left or right side. The subject must respond to the target immediately after detecting it on the left or right visual field. The side of target presentation is most often announced by a previous "cue" stimulus, which may be either "valid" (it appears on the correct target side) or "invalid" (it appears on the opposite side) [11]. This paradigm is used for assessing the engagement, disengagement, orientation, and covert shifting of attention. Some investigators propose the magnocellular system (M-system) as the neural basis of such deficits. This system processes the information, followed by sending the processed information into the posterior parietal cortex, which is a particular multi-modal selective spatial attention area. The M-system is crucial for smoothing the flow of attention, which plays an important role for the identification of individual letters in a text. In addition, the automatisation deficit hypothesis is in line with the M-system deficit, but it attributes that abnormal spatial and temporal processing to the output from the cerebellum rather than to the M-system input only [5].

Neurofeedback is one of the applications of Brain-computer interface (BCI) in rehabilitation and behavioral medicine [12]. Neurofeedback is reportedly used to treat associated deficits in children with reading disability [13] and also some of other psychiatric disorders [14] [15]. Some studies reported improvements in the reading ability and phonological awareness deficit, as well as changes in the EEG in children with reading disabilities as a result of neurofeedback in a single-subject study [16].

The theoretical basis of neurofeedback is based on a biological model of Learning Disorders (LD), considering that $\mathrm{LD}$ is a disorder of neural regulation and assuming under this approach that these neural deficiencies are amenable to change using the feedback method (e.g. an operant conditioning procedure, whereby an individual learns to self-regulate the electrical activity) [17].

A protocol of neurofeedback that introduced by Hammond (2005) was used in this study. This protocol directly or indirectly affected the cerebellum and occipital areas interfering in visual process and movement anal- 
ysis [18]. Accordingly, it was decided to use protocol to evaluate visouspatial attention ability by means of Posner paradigm task and ERP in children with reading disabilities.

\section{Methods}

\subsection{Participants}

Participants were chosen from children aged 8 - 12, whom were attending the Learning Disorder Center ${ }^{1}$ in Tehran. Children from three different Learning Disorder Centers ${ }^{1}$ were selected by convenient sampling method. 18 parents agreed to participate in the study. Inclusion criteria included: 1) having the diagnosis of reading disability by a psychiatrist according to DSM-V criteria; 2) children without vision problems, or whom in which refractive errors have been resolved with the use of assistive devices; 3) being right handed and 4) having no history of mental disease, brain injury, neurological disorder, serious medical condition, and attention deficit hyperactivity disorder (ADHD) or other psychiatric disorder. Only 4 children with respect to inclusion criteria were entered in the study, during which 2 were excluded (due to personal reasons), having 2 children (2 males) remaining in the study. The study was explained to the parents of the participants, and a consent form was signed.

\subsection{Visual-Spatial Attention Task}

The Posner paradigm task was used for measuring visual-spatial attention ability (Figure 1). In this paradigm, children reacted with a spatially concordant motor response to left or right visual targets. The targets could be either preceded by a spatial cue ("valid" = same side; "invalid" = opposite side), or presented uncued [10]. The measured behavioral variables in this paradigm were Reaction Time (RT), correct answer, miss error, and anticipation error.

\subsection{Electroencephalographic Recording}

Continuous electroencephalogram was recorded by the Mitsar system (Mitsar ${ }^{\circledR}$, Russia) using 19 surface electrodes (Electro cap ${ }^{\circledR}$ ). The electrode positions included the international 10 - 20 System. All electrodes were referenced to link the ear lobes, with the ground electrode placed between the Fz and Fpz electrodes. Electrode impedance was maintained below $10 \mathrm{k}$ Ohm. EEG was sampled at $500 \mathrm{~Hz}$ with filtered online $0.1-50 \mathrm{~Hz}$ band pass.

\subsection{Waveform Analysis}

Movement artifacts were excluded from analysis by a careful visual inspection of the raw EEG. Moreover, ocular artifacts were corrected by applying the independent component analysis (ICA) method using the WinEEG software. Only the artifact-free and correct responses epochs are included in averaged ERPs. Data from trials were averaged according to the task conditions (Valid-right, Invalid-right, Valid-left and Invalid-left) over a window of $600 \mathrm{~ms}$ with $100 \mathrm{~ms}$ pre-stimulus and band-pass filtered between $0.1 \mathrm{~Hz}$ and $30 \mathrm{~Hz}$, band-pass filter.

\subsection{Neurofeedback Set}

ProComp Infiniti Encoder (Thought Technology Ltd.), and Biograph Infiniti software (V5.1.3), were used to give feedback for brain wave conditioning. The EEG recording sampling rate was $256 \mathrm{~Hz}$. The protocol which was used in this study was defined by Hammond (2005) as balance protocol. This protocol consists of a bipolar montage of two electrodes directly under electrode sites $\mathrm{O} 1$ and O2. A ground electrode was placed on the left earlobe. The participants were trained to inhibit 4 - $7 \mathrm{~Hz}$ while reinforcing 15 - $18 \mathrm{~Hz}$. The trainer, sat behind the participant, observing the information about the power in each of these frequency bands online on a monitor, while giving feedback audio-visually to the participant via a second monitor. Feedback consisted of negative feedback and reinforcement: whenever spectral amplitudes were above threshold for theta band $(4-7 \mathrm{~Hz})$ or below threshold for beta band (15 - $18 \mathrm{~Hz}$ ), the software briefly interrupted the ongoing movie.

${ }^{1}$ Learning disorder centers 1 \& 2: jamalludin asad abady St, between farhang and kalantari square, Tehran, Iran Tel: +982188060329; Learning disorder center 3: Valiasr junction, north baradaran mozafar St, Tehran, Iran Tel: +982166413159. 


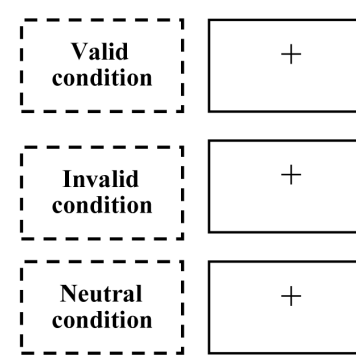

Fixation point

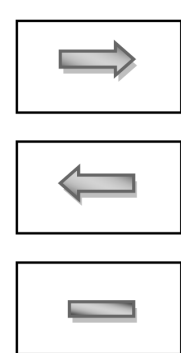

Cue
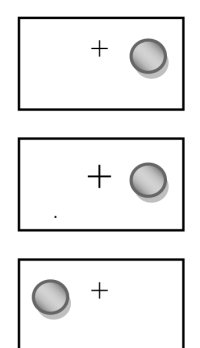

Target (circle)

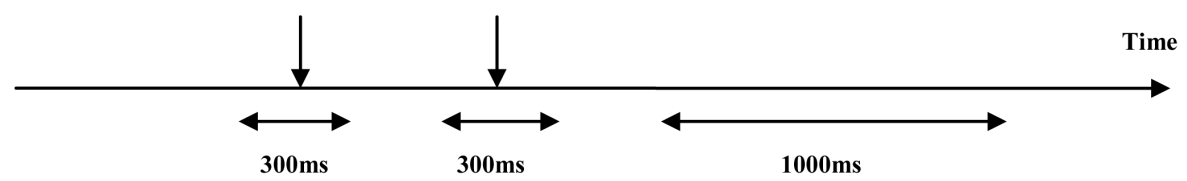

Figure 1. Outline of the experimental procedure depicting the sequence of events in each of the three experimental conditions. Cue: gray cue. Target: gray circle (on the same side in the valid condition, and on the opposite side in the invalid condition). The fixation point was a black cross in the middle of the screen.

\subsection{Procedure}

An ABA single subject design was used to conduct this study in the following phases; (phase A) pre-treatment phase as a baseline, (phase B) treatment; involved 12 sessions of neurofeedback twice a week, with each session lasting 30 minutes, and (phase C) post-treatment. Repeated measurements (Posner paradigm task) were performed throughout the baseline (4 sessions), treatment (12 sessions), and post treatment period (4 sessions), in order to evaluate the changes in visual-spatial attention ability of children. An ERP was also recorded before and after the treatment phase.

\subsection{Data Analysis}

The data were assessed by the visual inspection of the graphs. Like other single subject designs, there was no intergroup comparison. The means, standard deviation (SDs), and Cohen d were also calculated for the baseline, treatment, and post treatment phases. Cohen $d$ is used in power analysis in behavioral science. Cohen defines effect sizes as "small, $d=0.2$ ", "medium, $d=0.5$ ", and "large, $d=0.8$ " [19].

\section{Results}

\subsection{Visual-Spatial Attention Performance}

Figure 2 shows the ABA design used to assess changes in visual-spatial attention ability ((a) number of correct responses, (b) reaction time-valid cue, (c) reaction time-invalid cue) following the neurofeedback in 2 children, separately. No major fluctuations are present in the data at other time points, suggesting the results are due to the treatment. Figure 2(a) demonstrates a repeated pattern of increase in the correct responses following the treatment in two children. Figure 2(b) and Figure 2(c) demonstrate a reduction pattern in the reaction time at both valid and invalid cues. The scores showed the least amount of change at the post treatment assessments after discontinuing the treatment.

Tables 1-3 present the information about the correct responses, reaction time-valid cue and reaction timeinvalid cue in 2 participants, respectively: mean, SD, Cohen d value at baseline, treatment, and post treatment assessments.

The value of effect size for the baseline-treatment phase and the treatment-post treatment phase in the correct responses was calculated "Table 1". Cohen d was -0.56 and -0.3 in participant 1 and 2, respectively, indicating small and medium effect of treatment. In comparing with baseline-treatment phase, the value of effect size in treatment-post treatment phase was large (Cohen $\mathrm{d}>0.8$ ) for both of them. As demonstrated in Table 2 and Table 3 for the Reaction time factor, all the effect sizes (Cohen $d>0.8$ ) were large, except the effect size value in baseline-treatment phase for case 1 , indicating small effect (0.36). 


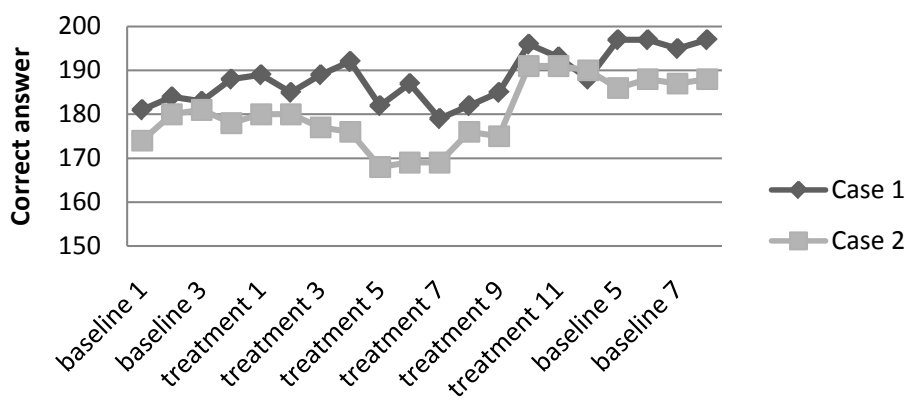

(a)

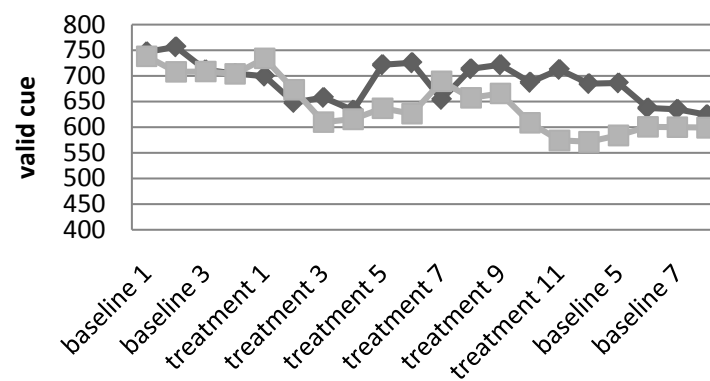

(b)
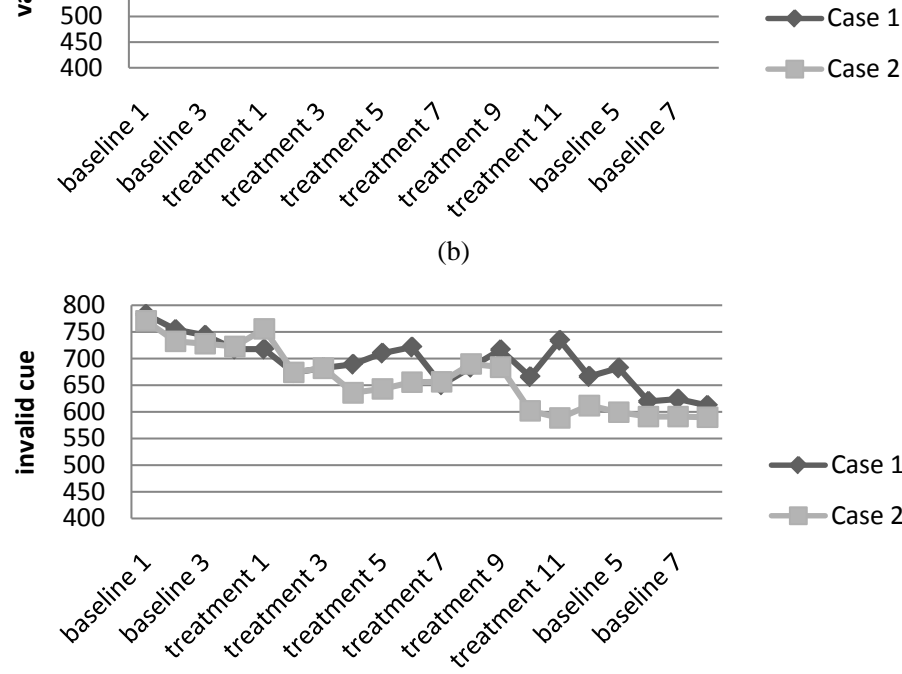

(c)

Figure 2. Pattern of visual-spatial attention ability changes across baseline, treatment and post treatment assessments conducted for 2 children: (a) Correct answers, (b) Reaction time-valid cue, (c) Reaction time-invalid cue.

Table 1. Correct responses at baseline, treatment and post treatment assessments. Data were expressed as mean and standard deviation (SD).

\begin{tabular}{ccccccccc}
\hline & Mean A1 & Mean B & Mean A2 & SD A1 & SD B & SD A2 & Cohen $d$ A1-B & Cohen $d$ B-A2 \\
\hline Case 1 & 192 & 188.33 & 196.33 & 6.74 & 6.32 & 0.94 & -0.56 & 1.77 \\
Case 2 & 180.75 & 178.5 & 187 & 5.88 & 8.44 & 1 & -0.3 & 1.4 \\
\hline
\end{tabular}

Abbreviations: A1; baseline, B; treatment, A2; post treatment, SD; standard deviation.

Table 2. Reaction time-valid cue at baseline, treatment and post treatment assessments. Data were expressed as mean and standard deviation (SD).

\begin{tabular}{ccccccccc}
\hline & Mean A1 & Mean B & Mean A2 & SD A1 & SD B & SD A2 & Cohen $d$ A1-B & Cohen $d$ B-A2 \\
\hline Case 1 & 701.18 & 683.83 & 640.33 & 48.93 & 46.2 & 32.52 & 0.36 \\
Case 2 & 714.63 & 634.17 & 592.31 & 13.71 & 53.98 & 8.4 & 1.59 \\
\hline
\end{tabular}

Abbreviations: A1; baseline, B; treatment, A2; post treatment, SD; standard deviation. 
Table 3. Reaction time-invalid cue at baseline, treatment and post treatment assessments. Data were expressed as mean and standard deviation (SD).

\begin{tabular}{ccccccccc}
\hline & Mean A1 & Mean B & Mean A2 & SD A1 & SD B & SD A2 & Cohen $d$ A1-B & Cohen $d$ B-A2 \\
\hline Case 1 & 712.1 & 678.66 & 642.11 & 45.3 & 43.5 & 28.69 & 0.75 & 0.99 \\
Case 2 & 725.58 & 642.75 & 595.25 & 31.97 & 56.24 & 4.2 & 1.81 & 1.19 \\
\hline
\end{tabular}

Abbreviations: A1; baseline, B; treatment, A2; post treatment, SD; standard deviation.

\subsection{Event-Related Potentials}

Figure 3 shows the grand average ERPs for both children in each of the four conditions (Valid-right, Invalidright, Valid-left and Invalid-left). As shown in Figure 3, two large major positive deflections elicited by Posner paradigm task over the parietal site, maximal at Pz (defined as the P3a and P3b components). The results demonstrated that the P3a and P3b amplitude was higher in baseline compared to post treatment, indicating a reduction in P3 components after neurofeedback training.

\section{Discussion}

The analysis of the behavioral data revealed an improvement in the visual-spatial attention ability by neurofeedback in the two participants. At the end of the treatment, the participants showed a reduction in the reaction time factor, and an increase in the correct responses. As demonstrated in the results, children showed a better performance on Posner paradigm task after treatment "Tables 1-3". Moreover, the post treatment assessments displayed stable behavioral improvements during the time. The improved behavioral performance was accompanied by P3a and P3b amplitude reduction after the EEG biofeedback training.

The efficacy of neurofeedback by normalization of targeted EEG abnormalities is reported in dyslexia, with improvements in the reading ability at least 2 grade levels post-neurofeedback [20]. Some researchers investigated the effects of neurofeedback on 12 children with reading disorder who were randomly assigned to experimental and control groups. After 20 treatment sessions, children in the experimental group showed significant improvement in spelling ability. However, no significant changes in brain mapping were shown [21]. Nazari et al. (2012) demonstrated that although EEG analysis did not show notable changes in the power of the targeted bands (delta, theta, and beta), there was normalization of coherence of the theta band at T3-T4, delta band at $\mathrm{Cz}-\mathrm{Fz}$, beta band at Cz-Fz, Cz-Pz and Cz-C4 [16].

The ERP parameters, such as amplitude and latency, are the indicators of brain functions. ERP is also related to the circumscribed cognitive processes. Predominantly, the P3 wave represents cognitive functions involved in orientation of attention, contextual updating, response modulation, and response resolution [9]. To explore the links between behavioral and EEG changes in the participants, the P3 component was analyzed. It has been demonstrated that deficits in later cognitive control and error monitoring mechanisms, indexed by the $\mathrm{P} 3$, are common in children with reading disorder. If ERP amplitude reflects brain activation level, increasing brain efficiency should be related to a decrease in the amplitude of ERP waves [22]. In this line, the results pointed out that after neurofeedback training the lower amplitude is related to more correct and less error response. This finding might be interpreted as a decreased attentional demand at neural level. Furthermore, P3 peak latency is proportional to stimulus evaluation timing, sensitive to task processing, and it varies with individual differences in cognitive capability [23]. We found shorter P3 latency after treatment, indicating an improvement in the timing of visouspatial processing.

The human visual system starts in the retina, and from the retina, visual information is relayed to the lateral geniculate nucleus and then onto the extra striate cortex (V1). Two distinct systems exist in visual pathway; the magnocellular or M-system, and the parvocellular or P-system. The M-system is sensitive to fast changing stimuli and moving objects and are insensitive to colors [24]. Some investigators propose the M-system as the neural basis of children with reading disorder. For example, Galaburda and Livingstone (1993), examined the brains of dyslexics post mortem, and found that the neurons in the magnocellular layers of the lateral geniculate nucleus were some $30 \%$ smaller than those of controls [25]. According to the magnocellular theory of dyslexia, impairments along the magnocelluar pathway would cause problems with reading [26]-[28]. The idea behind the role of the M-system in reading is that any weakness in M-system can cause visual confusion of letter order and 


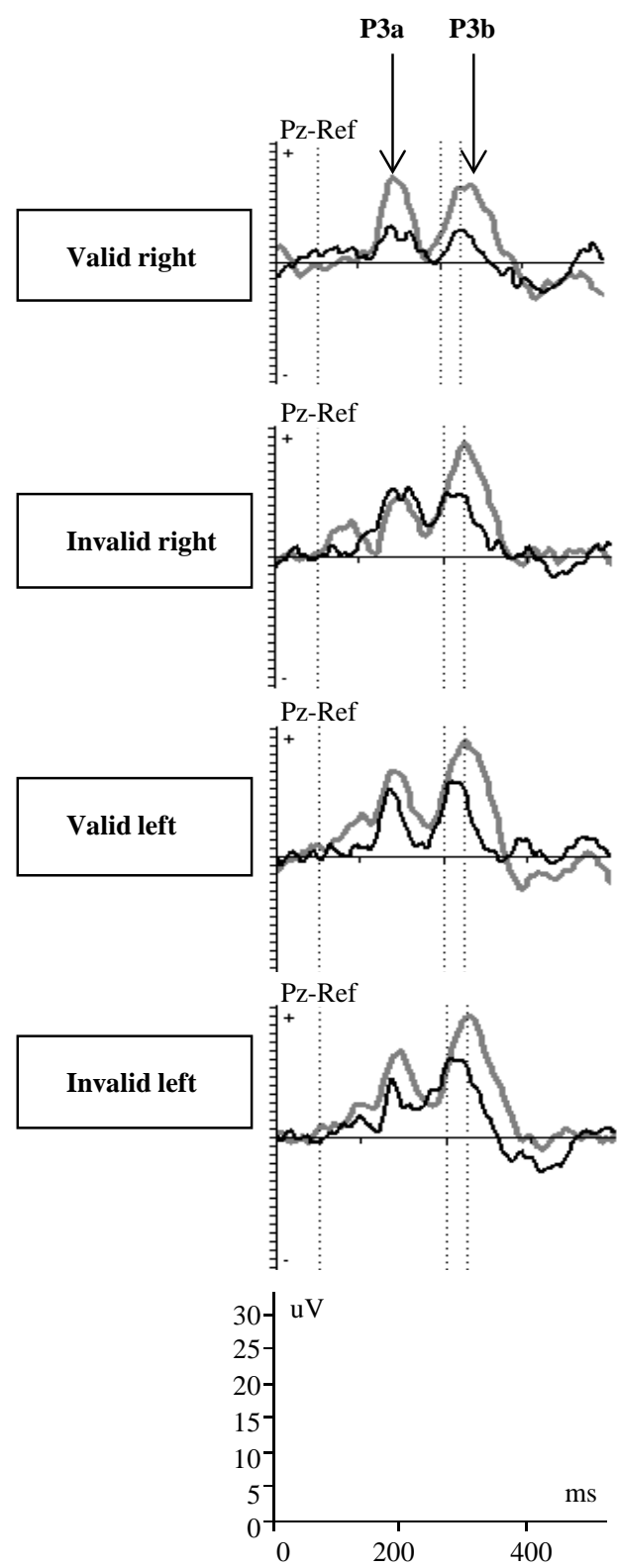

Figure 3. Grand average ERPs as a function of cue validity (valid, invalid) and target location (right, left), separately for baseline (gray line) and post treatment (black line).

poor visual memory for written word [29]. Considering the evidence about impaired functioning of the M-system in dyslexics and the projection of this system on the posterior parietal cortex, there are some evidence that the posterior parietal cortex is involved in directing visual-spatial attention [30], and it is also known that damage to the posterior parietal cortex affects the ability to disengage visual attention [31].

Cells in the primary visual cortex project further to the cerebellum and superior colliculus, which are important for visuo-motor control. On the other hand, it is known that cerebellar dysfunction impairs linguistic processing, acquisition and automatisation of new cognitive procedures [32], the reason why some researchers have proposed cerebellar theory of dyslexia [33]. Problems with balancing are other manifestations of cerebellar impairments that have also been found frequently in dyslexic children [33]. Nicolson \& Fawcett (1999) suggest that because the M-system projects onto the cerebellum, deviant functioning of the magnocelluar pathway could be responsible for cerebellar dysfunctions found in dyslexic children [34].

For neurofeedback purpose in this study, the balance protocol introduced by Hammond (2005) was used. It 
has been hypothesized that improving function in the vicinity of primary visual cortex (i.e. Brodmann area 17 - 18) may improve the visual guidance for the cerebellum. This protocol affects directly or indirectly on cerebellum and occipital areas interfering in visual process [18]. Because of the linkage between balancing, cerebellum, magnocelluar pathway, and visual-spatial attention in dyslexic children, the results would explain the improvements in visual-spatial attention performance at behavioral and neural level. However, further controlled studies with larger sample sizes seem merited to replicate these findings and to investigate the mechanism of action by which the positive results are obtained from dyslexic children.

\section{References}

[1] Sadock, B.J. and Sadock, V.A. (2009) Kaplan and Sadock’s Concise Textbook of Child and Adolescent Psychiatry. Wolters Kluwer Health, Philadelphia, PA.

[2] Barbiero, C., Lonciari, I., Montico, M., Monasta, L., Penge, R., Vio, C., et al. (2012) The Submerged Dyslexia Iceberg: How Many School Children Are Not Diagnosed? Results from an Italian Study. PLoS ONE, 7, e48082. http://dx.doi.org/10.1371/journal.pone.0048082

[3] Bosse, M.-L., Tainturier, M.J. and Valdois, S. (2007) Developmental Dyslexia: The Visual Attention Span Deficit Hypothesis. Cognition, 104, 198-230. http://dx.doi.org/10.1016/j.cognition.2006.05.009

[4] Bigler, E.D., Lajiness-O’Neill, R. and Howes, N.-L. (1998) Technology in the Assessment of Learning Disability. Journal of Learning Disabilities, 31, 67-82. http://dx.doi.org/10.1177/002221949803100107

[5] Facoetti, A., Luisa Lorusso, M., Paganoni, P., Umilta, C. and Gastone Mascetti, G. (2003) The Role of Visuospatial Attention in Developmental Dyslexia: Evidence from a Rehabilitation Study. Cognitive Brain Research, 15, $154-164$. http://dx.doi.org/10.1016/S0926-6410(02)00148-9

[6] Vidyasagar, T.R. and Pammer, K. (2010) Dyslexia: A Deficit in Visuo-Spatial Attention, Not in Phonological Processing. Trends in Cognitive Sciences, 14, 57-63. http://dx.doi.org/10.1016/j.tics.2009.12.003

[7] Vidyasagar, T.R. (2012) Aetiology of Dyslexia: A Visual Perspective on a Phonological Marker. In: Stein, J. and Kapoula, Z., Eds., Visual Aspects of Dyslexia, Oxford University Press, Oxford, 151.

[8] Vidyasagar, T.R. (2004) Neural Underpinnings of Dyslexia as a Disorder of Visuo-Spatial Attention. Clinical and Experimental Optometry, 87, 4-10. http://dx.doi.org/10.1111/j.1444-0938.2004.tb03138.x

[9] Sanei, S. and Chambers, J.A. (2008) EEG Signal Processing. John Wiley \& Sons Ltd., Hoboken.

[10] Perchet, C., Revol, O., Fourneret, P., Mauguière, F. and Garcia-Larrea, L. (2001) Attention Shifts and Anticipatory Mechanisms in Hyperactive Children: An ERP Study Using the Posner Paradigm. Biological Psychiatry, 50, 44-57. http://dx.doi.org/10.1016/S0006-3223(00)01119-7

[11] Posner, M.I. (1980) Orienting of Attention. Quarterly Journal of Experimental Psychology, 32, 3-25. http://dx.doi.org/10.1080/00335558008248231

[12] Allanson, J. and Fairclough, S.H. (2004) A Research Agenda for Physiological Computing. Interacting with Computers, 16, 857-878. http://dx.doi.org/10.1016/j.intcom.2004.08.001

[13] Othmer, S., Othmer, S.F. and Marks, C.S. (1991) EEG Biofeedback Training for Attention Deficit Disorder, Specific Learning Disabilities, and Associated Conduct Problems. EEG Spectrum. http://www.eegspectrum.com/Applications/ADHD-ADD/

[14] Kim, J.I., Yoon, S., Oh, H.K. and Lee, S.-H. (2015) Clinical Significance for Neurofeedback Training of Children with Attention-Deficit/Hyperactivity Disorder. Journal of Korean Neuropsychiatric Association, 54, 62-68.

[15] van Schie, H.T., Gerrits, B.J., Buitelaar, J.K. and Kouijzer, M.E.J. (2014) EEG Biofeedback for Autism Spectrum Disorders: A Reply to a Commentary by Coben and Ricca (2014). Applied Psychophysiology and Biofeedback, 40, 57-60. http://dx.doi.org/10.1007/s10484-015-9272-2

[16] Nazari, M.A., Mosanezhad, E., Hashemi, T. and Jahan, A. (2012) The Effectiveness of Neurofeedback Training on EEG Coherence and Neuropsychological Functions in Children with Reading Disability. Clinical EEG and Neuroscience, 43, 315-322. http://dx.doi.org/10.1177/1550059412451880

[17] Butnik, S.M. (2005) Neurofeedback in Adolescents and Adults with Attention Deficit Hyperactivity Disorder. Journal of Clinical Psychology, 61, 621-625. http://dx.doi.org/10.1002/jclp.20124

[18] Hammond, D.C. (2005) Neurofeedback to Improve Physical Balance, Incontinence, and Swallowing. Journal of Neurotherapy, 9, 27-36. http://dx.doi.org/10.1300/J184v09n01_03

[19] Becker, L.A. (2006) Effect Size (ES). http://www2.jura.uni-hamburg.de/instkrim/kriminologie/Mitarbeiter/Enzmann/Lehre/StatIIKrim/EffectSizeBecker.pdf

[20] Walker, J.E. and Norman, C.A. (2006) The Neurophysiology of Dyslexia: A Selective Review with Implications for 
Neurofeedback Remediation and Results of Treatment in Twelve Consecutive Patients. Journal of Neurotherapy, 10, 45-55. http://dx.doi.org/10.1300/J184v10n01_04

[21] Breteler, M.H.M., Arns, M., Peters, S., Giepmans, I. and Verhoeven, L. (2010) Improvements in Spelling after QEEGBased Neurofeedback in Dyslexia: A Randomized Controlled Treatment Study. Applied Psychophysiology and Biofeedback, 35, 5-11. http://dx.doi.org/10.1007/s10484-009-9105-2

[22] Robaey, P., Cansino, S., Dugas, M. and Renault, B. (1995) A Comparative Study of ERP Correlates of Psychometric and Piagetian Intelligence Measures in Normal and Hyperactive Children. Electroencephalography and Clinical Neurophysiology/Evoked Potentials Section, 96, 56-75. http://dx.doi.org/10.1016/0013-4694(94)00174-J

[23] Polich, J. (2007) Updating P300: An Integrative Theory of P3a and P3b. Clinical Neurophysiology, 118, $2128-2148$. http://dx.doi.org/10.1016/j.clinph.2007.04.019

[24] Palmer, S.E. (1999) Vision Science: Photons to Phenomenology. MIT Press, Cambridge.

[25] Galaburda, A. and Livingstone, M. (1993) Evidence for a Magnocellular Defect in Developmental Dyslexia. Annals of the New York Academy of Sciences, 682, 70-82. http://dx.doi.org/10.1111/j.1749-6632.1993.tb22960.x

[26] Stein, J. (1997) To See but Not to Read; The Magnocellular Theory of Dyslexia. Trends in Neurosciences, 20, $147-152$. http://dx.doi.org/10.1016/S0166-2236(96)01005-3

[27] Stein, J. (2001) The Sensory Basis of Reading Problems. Developmental Neuropsychology, 20, 509-534. http://dx.doi.org/10.1207/S15326942DN2002_4

[28] Stein, J. (2001) The Magnocellular Theory of Developmental Dyslexia. Dyslexia, 7, 12-36. http://dx.doi.org/10.1002/dys.186

[29] Boden, C. and Giaschi, D. (2007) M-Stream Deficits and Reading-Related Visual Processes in Developmental Dyslexia. Psychological Bulletin, 133, 346-366. http://dx.doi.org/10.1037/0033-2909.133.2.346

[30] Kinsey, K., Rose, M., Hansen, P., Richardson, A. and Stein, J. (2004) Magnocellular Mediated Visual-Spatial Attention and Reading Ability. NeuroReport, 15, 2215-2218. http://dx.doi.org/10.1097/00001756-200410050-00014

[31] Posner, M.I. and Cohen, Y. (1984) Components of Visual Orienting. In: Bouma, H. and Bouwhuis, D.G., Eds., Attention and Performance X: Control of Language Processes, Erlbaum, Hillsdale, 531-556.

[32] Molinari, M., Leggio, M.G., Solida, A., Ciorra, R., Misciagna, S., Silveri, M.C. and Petrosini, L. (1997) Cerebellum and Procedural Learning: Evidence from Focal Cerebellar Lesions. Brain, 120, 1753-1762. http://dx.doi.org/10.1093/brain/120.10.1753

[33] Nicolson, R.I., Fawcett, A.J. and Dean, P. (2001) Developmental Dyslexia: The Cerebellar Deficit Hypothesis. Trends in Neurosciences, 24, 508-511. http://dx.doi.org/10.1016/S0166-2236(00)01896-8

[34] Nicolson, R.I. and Fawcett, A.J. (1999) Developmental Dyslexia: The Role of the Cerebellum. Dyslexia, 5, $155-177$. http://dx.doi.org/10.1002/(SICI)1099-0909(199909)5:3<155::AID-DYS143>3.0.CO;2-4 